Editorial

\title{
La imagen anatomo-metabólica PET-CT en el carcinoma de origen desconocido
}

Desde su introducción en la práctica clínica en 2001 (von Schulthess et al.University Hospital of Zurich. 2001), la combinación de la imagen anatómica (CT) y metabólica (PET) gracias a los equipos híbridos y a las estaciones de fusión ha supuesto una auténtica revolución dentro del diagnóstico por la imagen, especialmente en el paciente oncológico ${ }^{1}$. La imagen PET-CT ha permitido hacer realidad algo largamente anhelado dentro del mundo del diagnóstico: fusionar dos especialidades complementarias como son la Medicina Nuclear (PET) y la Radiología (CT). El resultado ha sido la obtención de una herramienta diagnóstica que suma lo aportado por la anatomía y el metabolismo, permitiendo aumentar la eficacia de ésta última al disminuir, en un porcentaje que oscila entre un 20-30\%, los falsos negativos y positivos que tradicionalmente se observan en la literatura publicada sobre la PET en Oncología ${ }^{2}$. Se trata en definitiva de mejorar el rendimiento diagnóstico de ambas exploraciones por separado, lo que redunda en un mejor abordaje de la enfermedad tumoral con lo que ello conlleva para la supervivencia.

Tanto la Sanidad Pública como Privada han apostado claramente por la imagen PET-CT. Los recientes informes sobre su utilidad clínica publicados tanto por el Ministerio de Sanidad y Consumo así como por la Comunidad de Madrid ${ }^{3,4}$ así lo demuestran. En el momento actual, 7 Comunidades Autónomas disponen de cámaras PET-CT, estando previsto que para el período 2006-07 esta cifra llegue hasta 10. Desde hace ya 2 años, la renovación/incorporación de equipos PET en ambas redes hospitalarias se hace, bien con cámaras PET-CT, o con estaciones de trabajo que permiten la fusión no sólo PET-CT sino también PET-RM. Precisamente, el equipo de investigadores que desarrolló la primera cámara PET-CT (Townsend et al,; University of Tennessee Medical Center. 1998) está trabajando en el nuevo equipo híbrido PET-RM que supondrá un nuevo paso en el mundo de la imagen diagnóstica en los próximos años.

Dentro de las indicaciones clínicas PET sometidas a Uso Tutelado por parte del Ministerio de Sanidad y Consumo desde Junio 2002 se incluye la identificación del tumor primario de origen desconocido en pacientes con metástasis de origen desconocido sin evidencia de tumor con las técnicas diagnósticas convencionales y que sean susceptibles de tratamiento radical. Curiosamente esta situación clínica no es contemplada por Medicare en Estados Unidos.

Tal y como se comenta en el artículo publicado en este número por la Dra. Cantos ${ }^{5}$, existe abundante bibliografía sobre la utilidad de la PET con FDG en esta situación clínica. Sin embargo, en la mayoría de los artículos se hace referencia a su baja especificidad por la existencia de falsos negativos y positivos, secundarios a la falta de referencias anatómicas propias de una exploración puramente metabólica como es la PET. El informe recientemente emitido por la Agencia de Evaluación de Tecnologías Sanitarias con los resultados del Uso Tutelado en la población española ${ }^{6}$, pone también de manifiesto la baja especificidad (80\%) en la detección de tumores primarios en relación al alto número de falsos positivos, frente a su mayor sensibilidad (83\%). La introducción de la imagen PET-CT podría suponer una mejora en esos resultados al disminuir la incertidumbre diagnóstica.

En los últimos meses han empezado a publicarse los primeros resultados de la PET-CT en esta situación clínica. Gutzeit et $\mathrm{al}^{7}$ del grupo del Hospital Universitario de Essen, reportan en una serie de 45 casos, la detección del tumor primario en el 33\% de los casos frente al 24\% de la PET y al 18\% de la CT. Nanni et al ${ }^{8}$ en una serie menos extensa (21 casos) refieren que la PET-CT detectó el primario en el 57\% de las situaciones frente al 24-40\% habitualmente publicado para la PET. Pendiente queda la aparición de los primeros resultados en pacientes de nuestro entorno, máxime tras la progresiva incorporación de las cámaras PET-CT en la red pública (Hospital La Paz, 2003). 
Dado que la PET-CT es una tecnología con apenas 5 años de existencia, son necesarias series más amplias que confirmen la mejora en el rendimiento diagnóstico que ofrece frente a la PET y la CT por separado. Si embargo, la bibliografía existente empieza a confirmar las importantes ventajas diagnósticas que supone su aplicación en el paciente oncológico ${ }^{9}$, especialmente si se usa globalmente (CT diagnóstico, PET diagnóstico y fusión de ambos) y no parcialmente (CT no diagnóstico para localización anatómica y PET diagnóstico) como ocurre en nuestro entorno sanitario.

\section{A. Maldonado, J. González Alenda Centro PET Recoletas La Milagrosa \\ Madrid}

\section{Bibliografía}

1. P J Ell. The contribution of PET/CT to improved patient management. British Journal of Radiology .2006; 79, 32-36.

2. Juweid ME, Cheson BD. PET and assessment of cancer therapy. N Eng J Med 2006; 354:496-507.

3. Rodríguez M, Asensio C, Maldonado A, et al. PET-TAC: Indicaciones, revisión sistemática y metaanálisis. Ministerio de Sanidad y Consumo. Instituto de Salud Carlos III. Agencia de Evaluación de Tecnologías Sanitarias (AETS). Madrid, Junio de 2004. ISBN: 84-95463-25-3.

4. Andradas E, Reza M, Gómez N, et al. Efectividad, seguridad e indicaciones del sistema híbrido PET/TAC. Informe Técnico IT01/2004. Unidad de Evaluación de Tecnologías Sanitarias. Agencia Laín Entralgo. Octubre 2004.

5. Cantos B, Sánchez A, Maximiano C, Hurtado A, Sánchez MR. Carcinoma de origen desconocido: diagnóstico y manejo terapéutico. Oncología 2006; 29(3):95-106.

6. Rodríguez M, Asensio C. Uso Tutelado de la PET con FDG. Ministerio de Sanidad y Consumo. Instituto de Salud Carlos III. Agencia de Evaluación de Tecnologías Sanitarias (AETS). Madrid, Noviembre de 2005. ISBN: 84-95463-30-X.

7. Gutzeit A, Antoch G, Kuhl H, et al. Unknown Primary Tumors: Detection with Dual-Modality PET/CT-Initial Experience. Radiology 2005 Nov 234:227-34.

8. Nanni C, Rubello D, Castellucci P, et al. Role of (18)F-FDG PET-CT imaging for the detection of an unknown primary tumour: preliminary results in 21 patients. Eur J Nucl Med Mol Imaging 2005 Feb 22.

9. von S, Steinert HC, Hany TF. Integrated PET/CT: current applications and future directions. Radiology. 2006 Feb;238(2):405-22. 